\title{
Modélisation du volume du fût d'arbre pour une gestion durable des écosystèmes forestiers soudaniens
}

\section{Noël Houédougbé FonTON ${ }^{1}$ Charles Chaffra YABI ${ }^{2}$ Jean Zinsou DAH-Dovonon ${ }^{2}$ Fortuné Kokou ADo Kо ${ }^{1}$ Titilayo Dотснамо ${ }^{1}$ \\ 1 Université d'Abomey-Calavi 01BP 526, Cotonou}

Bénin

2 Direction générale des forêts et des ressources naturelles

Cotonou

Bénin

La difficulté de disposer d'un tarif de cubage de chaque essence en forêt naturelle a conduit les aménagistes à utiliser le coefficient de forme proposé par Dawkins. Cette note propose une méthode conciliant les exigences écologiques (aspect non destructif du cubage des arbres sur pied) et la mise à disposition d'outils adéquats d'estimation du volume des arbres sur pied. Trois essences ont été choisies dans la forêt classée de Wari-Maro au Bénin pour mener l'étude : Isoberlinia spp., Anogeissus leiocarpa et Daniellia oliveri.

Il ressort que la meilleure équation de cubage du volume du fût est de la forme $V=a+b D^{2} H$ quelle que soit l'essence. L'étude comparative révèle une amélioration de l'estimation du volume fût de l'ordre de 10 \% sur le modèle de Dawkins.

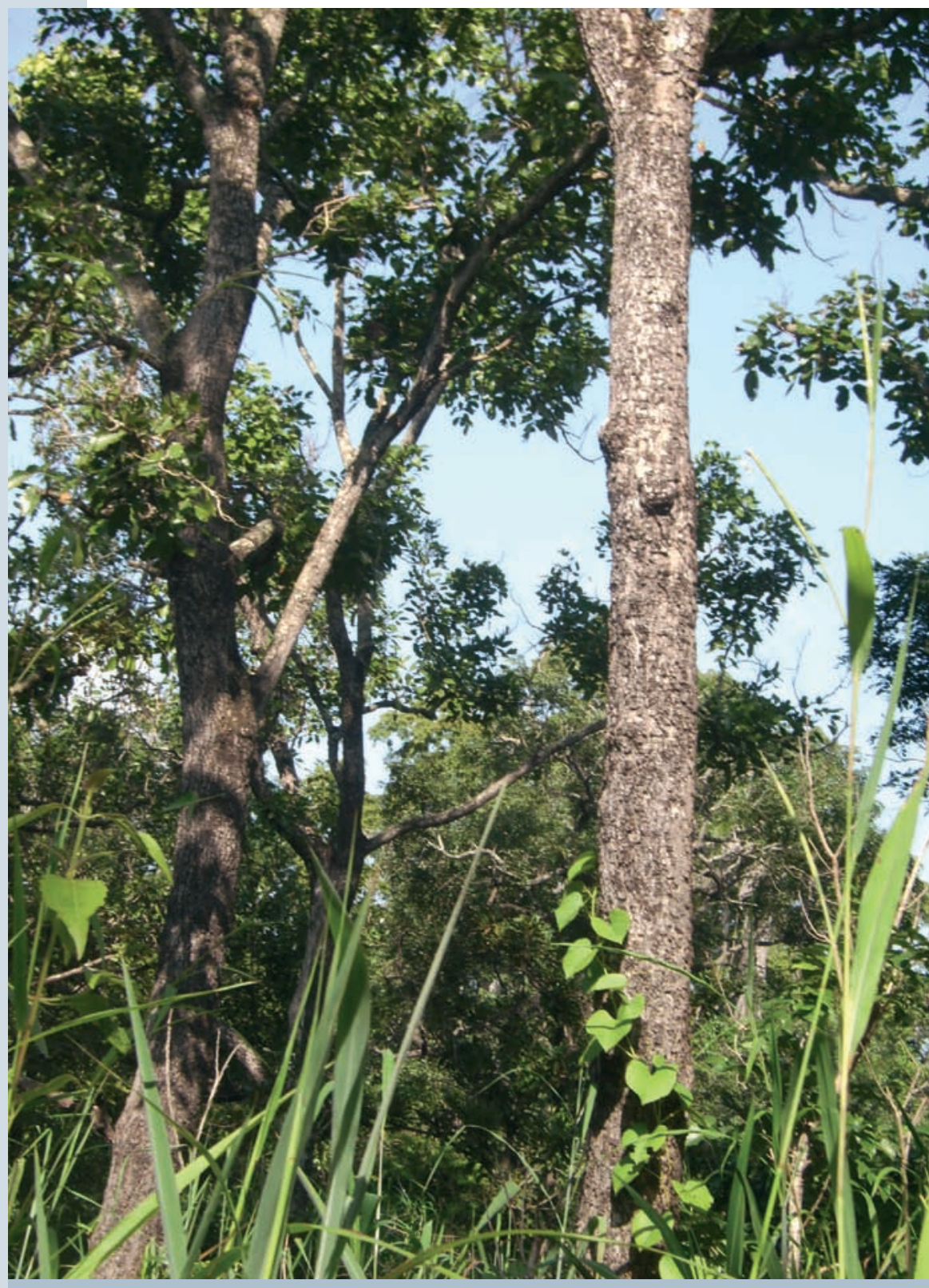

Photo 1.

Pied d'Isoberlinia doka dans la forêt classée de Wari-Maro, Bénin. Photo C. C. Yabi. 


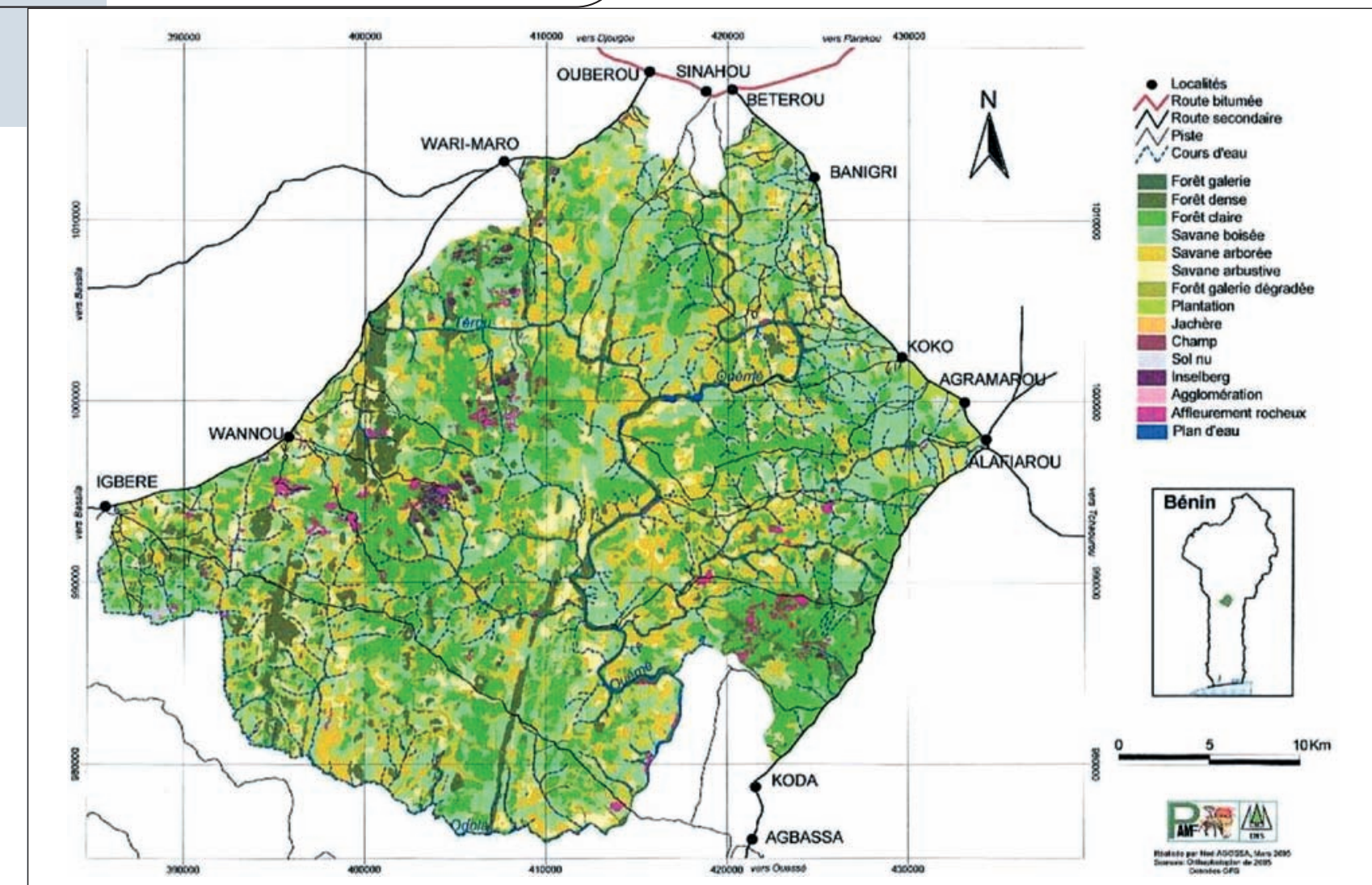

Figure 1.

Occupation du sol de la forêt classée de Wari-Maro, Bénin.

\section{Introduction}

La gestion durable des ressources forestières requiert des collectes de données adéquates (AKInSAnMI, AKIndéLÉ, 1995). Ces actions permettent à l'aménagiste de disposer d'indicateurs sur le potentiel ligneux afin d'élaborer un plan d'aménagement conséquent. La conjonction de l'impact considérable de l'homme sur son environnement au cours de ces cinquante dernières années et des conditions statistiques pour la qualité des modèles justifient les scénarios envisagés pour le futur dans le cadre de l'évaluation des écosystèmes pour le millénaire, dont la modélisation des volumes des arbres sans abattage. En effet, l'abattage des arbres pour l'élaboration des tarifs de cubage ne permet pas une approche de gestion des ressources naturelles visant à répondre aux besoins à la fois écologiques et humains futurs.

Dès lors, il faut mettre en œuvre des actions pour limiter les conséquences néfastes de la dégradation des écosystèmes avec l'utilisation des coefficients de forme proposés par DAWKINS (1961). Pour cet auteur, la valeur du coefficient de forme des essences tropicales est de 0,55 pour l'estimation du volume total et de 0,70 pour le volume du fût. Ces valeurs ne sont pas en adéquation avec la morphologie des arbres en zone soudanienne.

Cette étude s'inscrit dans une approche méthodologique d'estimation de volume du fût pour Isoberlinia spp. (Isoberlinia doka et Isoberlinia tomentosa), Anogeissus leiocarpa et Daniellia oliveri (photos 1, 2 et 3).

\section{Matériel et méthodes Milieu et données d'étude}

La forêt classée de Wari-Maro, située en zone soudanienne au Bénin, a servi à la collecte des données. D’une superficie totale de 107500 ha, elle est comprise entre $8^{\circ} 80^{\prime}$ et $9^{\circ} 10^{\prime}$ de latitude Nord et $1^{\circ} 55^{\prime}$ et $2^{\circ} 25^{\prime}$ de longitude Est et se caractérise par des peuplements de forêts claires et de savanes (figure 1) à dominance Isoberlinia. Ces peuplements sont établis sur des sols ferrallitiques et ferrugineux tropicaux. Le climat est de type soudanien humide avec une saison pluvieuse et une saison sèche dont les durées sont presque égales. La pluviosité moyenne annuelle est de $1152 \mathrm{~mm}$ et la température moyenne de $26,9^{\circ} \mathrm{C}$.

Les arbres échantillons sont de diamètre à 1,30 m (Dbh) supérieur ou égal à $10 \mathrm{~cm}$. Ils sont répartis dans six classes de diamètre d'amplitude 10 et dont la dernière est ouverte (60 cm et plus). Après avoir mesuré le Dbh, les données de cubage sont établies par des mesures faites avec le ruban diamètre et le Relascope de Bitterlich.

Au ruban, les diamètres souche à $0,10 \mathrm{~m}$ du sol $\left(D_{s}\right)$, à $0,5 \mathrm{~m}\left(\mathrm{D}_{0,5 \mathrm{~m}}\right)$ et à $1,5 \mathrm{~m}\left(\mathrm{D}_{1,5 \mathrm{~m}}\right)$ sont pris. Avec le Relascope et à une distance stationnement de $15 \mathrm{~m}$, les diamètres à $2 \mathrm{~m}$ $\left(D_{2}\right)$, à $3 \mathrm{~m}\left(\mathrm{D}_{3} \mathrm{~m}\right)$, à $4 \mathrm{~m}\left(\mathrm{D}_{4} \mathrm{~m}\right)$ et au sommet du fût $\left(\mathrm{D}_{\mathrm{Hf}}\right)$ sont déterminés par équivalence des bandes (figure 2). Ensuite, les hauteurs totale $\left(\mathrm{H}_{\mathrm{t}}\right)$ et fût $\left(\mathrm{H}_{\mathrm{f}}\right)$ sont mesurées.

La problématique du cubage des billons repose sur le principe de la révolution d'un solide (PHILIP, 1998). Dans cette logique, la formule de Hubert, de Smalian et celle du tronc de cône sont comparées pour déterminer le type dendromé- 


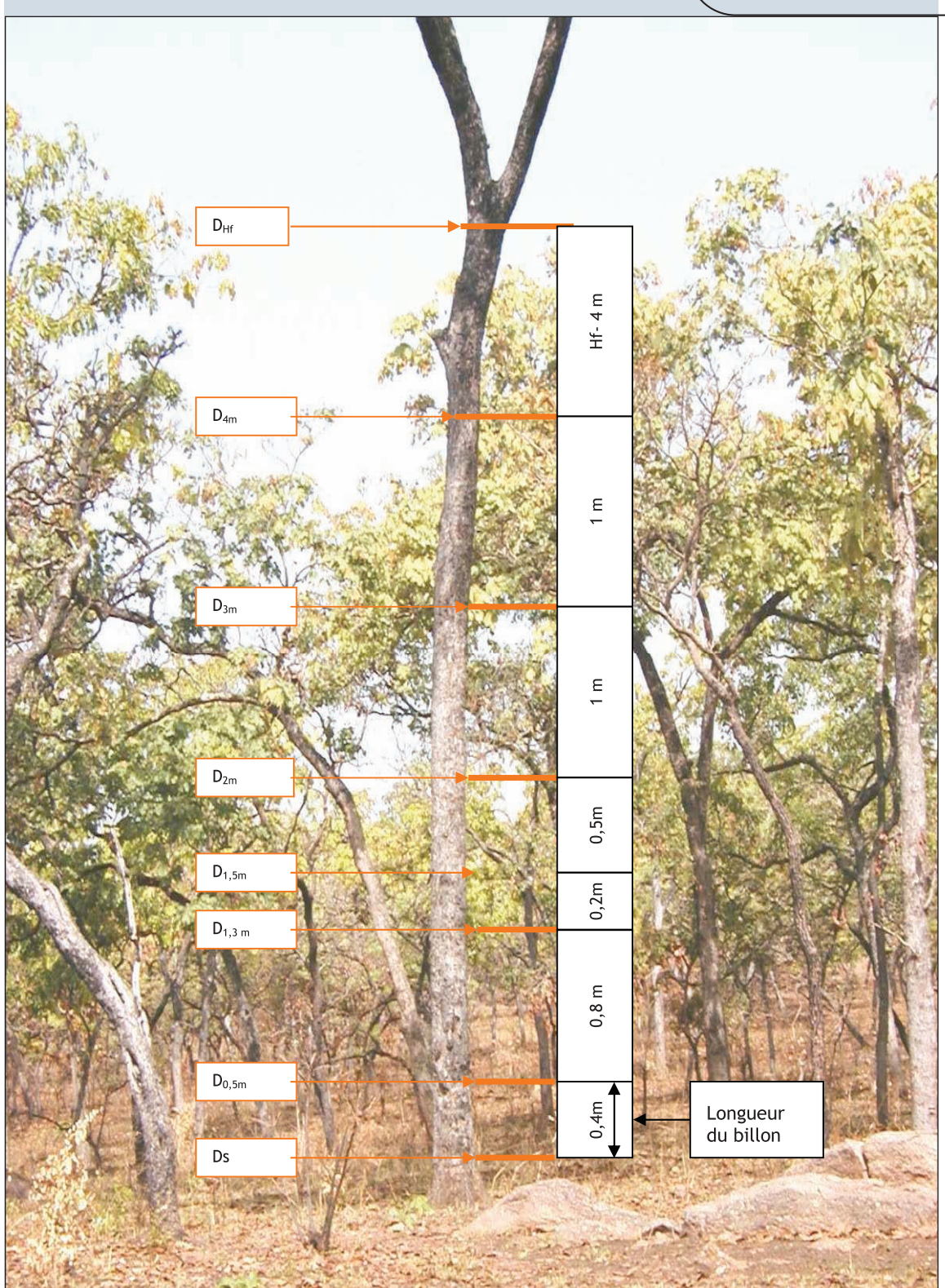

Figure 2.

Matérialisation des mesures d'arbres sur pied à différents niveaux d'une tige.

\section{Modèles de tarif de cubage} comparés et critères de choix

Neuf modèles de tarif de cubage sont comparés :

Modèle $1: V=a_{o}+a_{1} D^{2}$

Modèle $2: V=a_{o}+a_{1} D+a_{2} D^{2}$

Modèle 3 :

$V=a_{o}+a_{1} H+a_{2} D^{2} H$

Modèle $4: V=a_{0}+a_{1} D^{2} H$

Modèle $5: \frac{V}{D^{2}}=a_{o}+a_{1} H$

Modèle 6 :

$V=a_{o}+a_{1} D^{2}+a_{2} H^{2}+a_{3} H^{2} D$

Modèle 7 :

$L_{n} V=a_{o}+a_{1} L_{n} D+a_{2} L_{n} H$

Modèle 8 :

$V=a_{0}+a_{1} D^{2}+a_{2} H^{2} D$

Modèle 9 :

$V=a_{0}+a_{1} H^{2}+a_{2} H^{2} D$ trique le mieux adapté au profil des arbres. Le critère de choix est l'écart, par catégorie de billons, au volume moyen des trois formules.

\section{Étude de forme des arbres}

Les coefficients de forme ont été calculés, soit :

$f=\frac{V_{f}}{G H_{f}}$

avec $V_{f}$ le volume réel du fût, $G$ la surface terrière à $1,30 \mathrm{~m}$ du sol et $H_{f}$ la hauteur du fût.

Les valeurs moyennes par classe de grosseur sont comparées au coefficient de forme du fût de Dawkins par un test de conformité d'une moyenne.
Dans ces modèles, $a_{i}(i=0,1,23)$ sont des coefficients de régression, $V$ est le volume du fût de l'arbre en $\mathrm{dm}^{3}$, $D$ est le $\mathrm{Dbh}$ en $\mathrm{cm}$ et $H$ est la hauteur du fût en $\mathrm{m}$. La méthode des moindres carrés est utilisée pour l'estimation des $a_{i}$.

Le choix du meilleur modèle est basé sur le coefficient de détermination $\left(R^{2}\right)$ et les moyennes quadratiques des résidus absolus $\left(S_{a}\right)$ et relatifs $\left(S_{r}\right)$ dont les expressions sont respectivement :

$$
S_{a}=\sqrt{\frac{1}{n} \sum_{i=1}^{n}\left(V_{i}-\hat{V}_{i}\right)^{2}} \text { et } S_{r}=\sqrt{\frac{1}{n} \sum_{i=1}^{n}\left(\frac{V_{i}-\hat{V}_{i}}{\hat{V}_{i}}\right)^{2}}
$$

L'examen des résidus avec la droite de Henry, le coefficient de corrélation entre les résidus standardisés et les scores normaux et le test de Shapiro et Wilk a été effectué. 


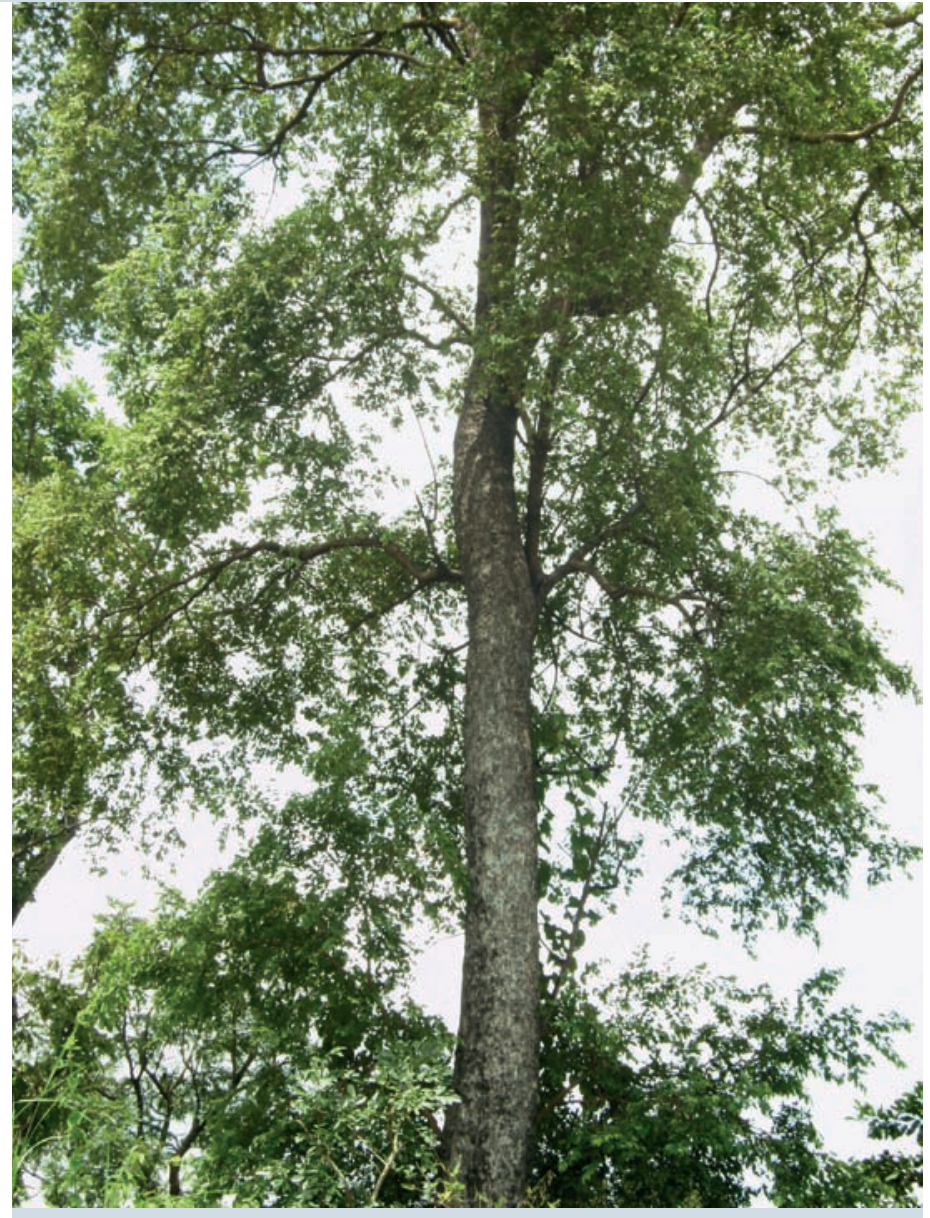

Photo 2.

Pied de Anogeissus leiocarpa dans la forêt classée de Wari-Maro, Bénin.

Photo C. C. Yabi.

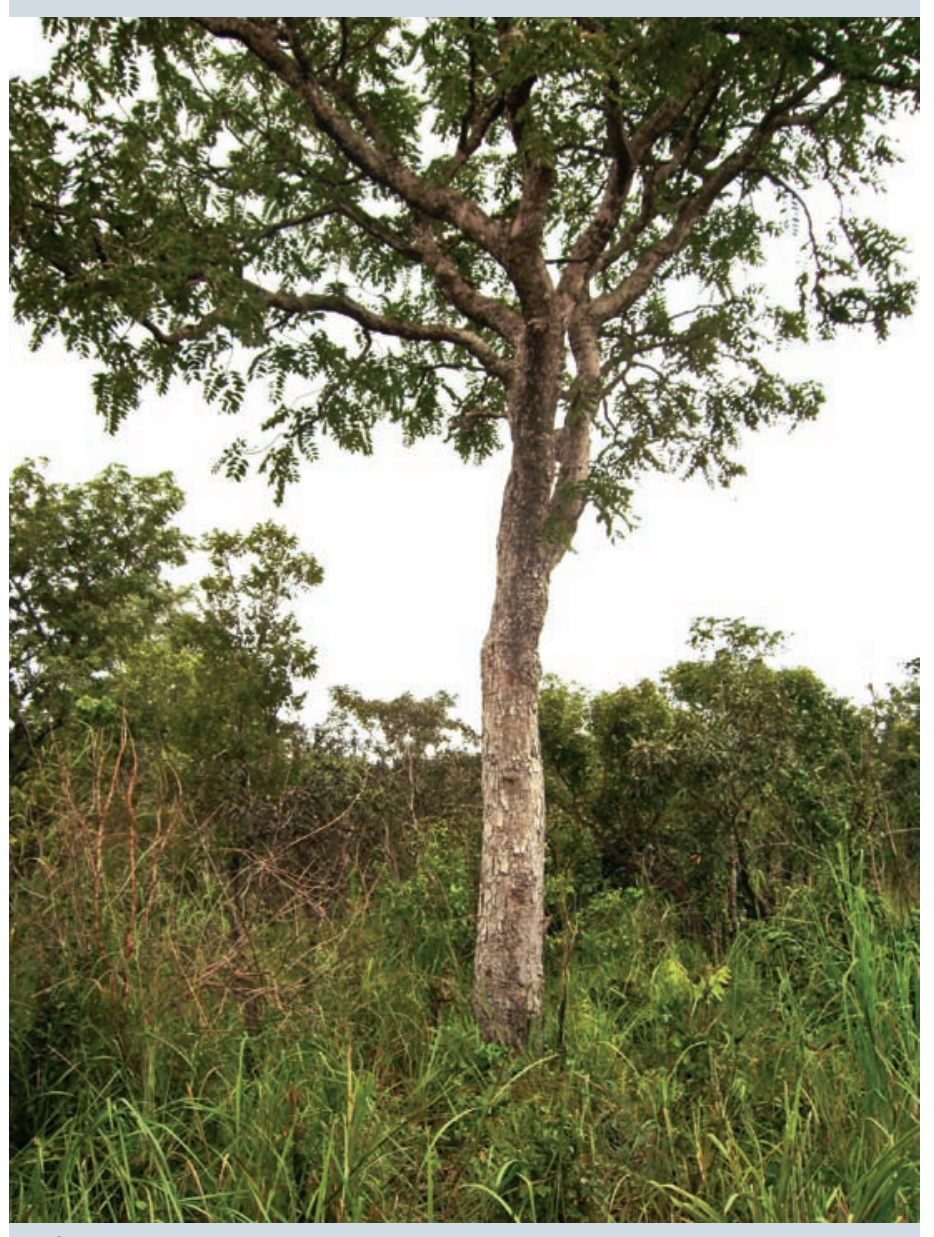

Photo 3.

Pied de Daniellia oliveri dans la forêt classée de Wari-Maro, Bénin.

Photo C. C. Yabi.

Tableau I.

Caractéristiques dendrométriques des arbres cubés sur pied.

\begin{tabular}{|c|c|c|c|c|}
\hline & & & & \\
\hline Essence & Caractéristiques & Moyenne & Minimum & Maximum \\
\hline Isoberlinia spp. (1 138 arbres) & Diamètre à 1,3 m (cm) & 31,1 & 10,0 & 60,0 \\
\hline & Hauteur totale (m) & 13,8 & 5,2 & 25,0 \\
\hline & Hauteur du fût (m) & 6,4 & 3,0 & 14,0 \\
\hline Anogeissus leiocarpa (600 arbres) & Diamètre à 1,3 m (cm) & 20,0 & 10,0 & 66,2 \\
\hline & Hauteur totale (m) & 16,4 & 8,2 & 28,6 \\
\hline & Hauteur du fût (m) & 7,1 & 2,0 & 15,2 \\
\hline Daniellia oliveri (400 arbres) & Diamètre à 1,3 m (cm) & 39,6 & 10,4 & 82,3 \\
\hline & Hauteur totale $(\mathrm{m})$ & 12,1 & 5,0 & 23,6 \\
\hline & Hauteur du fût (m) & 4,1 & 1,6 & 8,7 \\
\hline
\end{tabular}


Tableau II.

Valeurs des coefficients de forme (f) et des ${ }{ }_{\text {obs }}$ correspondantes par classe de grosseur et par essence.

\begin{tabular}{|l|l|r|r|}
\hline Essence & Classe de diamètre $(\mathrm{cm})$ & $\mathrm{f}$ & u \\
\hline Isoberlinia spp. & $10-19,9$ & 0,81 & 10,65 \\
\hline & $20-29,9$ & 0,83 & 28,27 \\
\hline & $30-39,9$ & 0,82 & 24,58 \\
\hline $40-49,9$ & 0,83 & 21,28 \\
\hline $50-59,9$ & 0,77 & 3,94 \\
\hline Anogeissus leiocarpa & 60 et plus & 0,79 & 2,81 \\
\hline & Total & 0,81 & 39,70 \\
\hline & $10-19,9$ & & \\
\hline $20-29,9$ & 0,91 & 22,79 \\
\hline $30-39,9$ & 0,84 & 15,40 \\
\hline & $40-49,9$ & 0,8 & 7,12 \\
\hline $50-59,9$ & 0,75 & 3,85 \\
\hline 60 et plus & 0,65 & 3,09 \\
\hline Total & 0,67 & 1,23 \\
\hline & & 0,77 & 15,59 \\
\hline
\end{tabular}

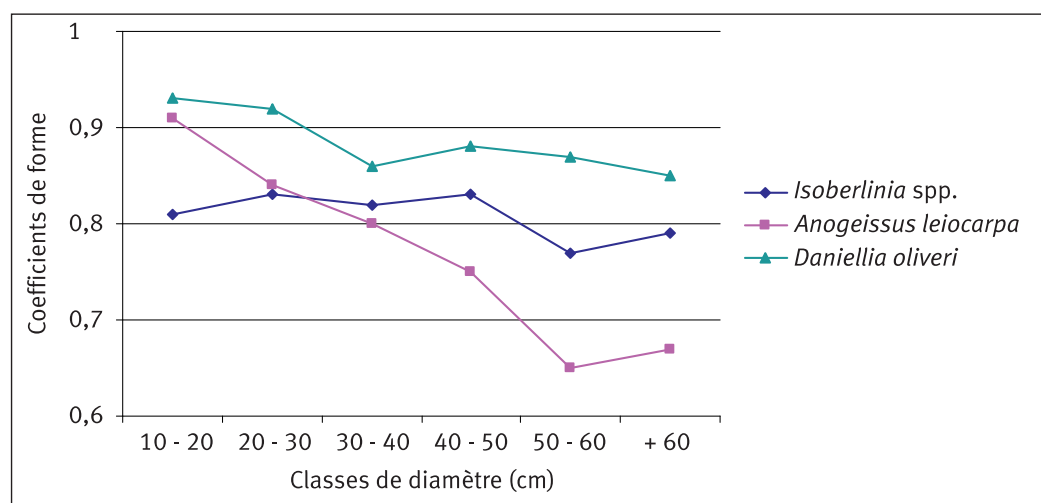

Figure 3.

Évolution du coefficient de forme en fonction de la grosseur de l'arbre.

\section{Résultats et discussion}

\section{Caractéristiques dendrométriques des arbres échantillons}

Le nombre d'arbres cubés est de 2138 avec 1138 pour Isoberlinia spp., 600 pour Anogeissus leiocarpa et 400 pour Daniellia oliveri. Comme le montre le tableau I, les Dbh varient entre $10 \mathrm{~cm}$ et $80 \mathrm{~cm}$, avec une moyenne de $31 \mathrm{~cm}, 20 \mathrm{~cm}$ et $40 \mathrm{~cm}$ respectivement pour Isoberlinia spp., Anogeissus leiocarpa et Daniellia oliveri. La valeur de la médiane montre que $50 \%$ des pieds possèdent un diamètre supérieur à $30 \mathrm{~cm}, 23 \mathrm{~cm}$ et $38 \mathrm{~cm}$ respectivement pour Isoberlinia spp., Anogeissus leiocarpa et Daniellia oliveri. En ce qui concerne la hauteur du fût, elle varie entre $1,6 \mathrm{~m}$ et $15 \mathrm{~m}$, et pour $50 \%$ des pieds elle ne dépasse pas $6 \mathrm{~m}$.

\section{Coefficient de forme des arbres}

Comme le montre la figure 3 , on peut admettre que la forme du fût est presque cylindrique pour les petits arbres. Les valeurs sont supérieures ou proches de 0,80 pour Isoberlinia spp. et Daniellia oliveri, alors que Anogeissus leiocarpa se caractérise par des coefficients de 0,80 pour des diamètres inférieurs à $40 \mathrm{~cm}$, pour décroître à 0,65 pour des diamètres supérieurs ou égaux à $40 \mathrm{~cm}$. La valeur relativement élevée pour Daniellia oliveri peut s'expliquer par la morphologie de la tige de cette espèce dans les forêts claires de la zone d'étude.

Les résultats du test de conformité mettent en évidence une différence significative entre les coefficients de forme calculés et la valeur de Dawkins, à l'exception des gros arbres pour Anogeissus. Pour tous les arbres (tableau II), $u_{\text {obs }}$ (valeur observée de la variable normale $u$ ) est égale à $39,7,15,6$ et 25,2 respectivement pour Isoberlinia spp., Anogeissus leiocarpa et Daniellia oliveri. On peut admettre globalement que les coefficients de forme sont différents de la valeur proposée par Dawkins. La valeur moyenne admissible pour les trois essences étudiées est de 0,80 , soit $10 \%$ de plus que celui de Dawkins. Cela repose le problème soulevé par THALMANN (1965), où les méthodes d'évaluation perfectionnées reposant sur des bases techniques confirmées sont nécessaires. 


\section{Établissement des équations de cubage du fût}

Les faibles valeurs des paramètres statistiques de validation sont obtenues avec la formule du tronc de cône. L'analyse graphique des résidus du modèle du tronc de cône fait ressortir une bonne répartition des résidus. En conséquence, l'hypothèse de la succession de troncs de cône le long de la tige de ces essences est retenue. Celle-ci est la plus raisonnable, car elle est moins tributaire de la longueur des billons et plus adaptée à la forme générale d'un arbre (PALM, 1982 ; PAuWels, RondeuX, 1999 ; Thibaut et al., 1998).

Les modèles d'estimation 3, 4, 6, 8 et 9 du volume du fût de Isoberlinia spp. sont caractérisés par $R^{2}$ supérieur à 0,93. Les faibles valeurs de $\left(S_{a}\right)$ et $\left(S_{r}\right)$ sont obtenues avec les modèles 3,4 et 6 . La droite de Henry présente une allure presque linéaire pour les modèles 3, 4 et 6 . L'histogramme des résidus présente une allure en cloche, caractéristique des distributions normales. Le coefficient de corrélation entre les résidus standardisés et les scores normaux est égal à 1 . Cette même démarche a été suivie pour la sélection des meilleurs modèles des deux autres essences. En matière d'équation de cubage, il est important de rester très attentif pour les petits arbres et pour les gros arbres. Les coefficients de corrélation entre les résidus standardisés et les scores normaux sont supérieurs à la valeur seuil de Ryan et Joiner qui est de 0,992 pour $\alpha$ égal à $5 \%$ (PALM, IEMMA, 2002). De plus, le test de normalité de Shapiro et Wilk confirme le caractère normal des résidus pour un niveau de signification de $5 \%$.

Le tarif de cubage fût est pour :

$$
\begin{array}{ll}
\text { Isoberlinia spp. } & V=13,0+0,062 D^{2} H \\
\text { Anogeissus leiocarpa } & V=10,5+0,0555 D^{2} H \\
\text { Daniellia oliveri } & V=18,8+0,0645 D^{2} H
\end{array}
$$

Les modèles ainsi obtenus, exprimant le volume en fonction du diamètre au carré $\left(D^{2}\right)$, sont les plus pertinents pour estimer le volume individuel des arbres (RONDEUX, 1999 ; LAUMANS, 1991 ; THIBAUt et al., 1998 ; Fonton et al., 2002).

\section{Conclusion}

La présente étude a permis d'éprouver une méthodologie d'établissement de tarif de cubage sans l'abattage des arbres.

Du point de vue descriptif, on note une sous-estimation de $10 \%$ du volume du fût avec le coefficient de forme proposé par Dawkins.

Du point de vue environnemental, l'aspect non destructif du cubage des arbres pour l'établissement des tarifs de cubage se révèle d'un intérêt certain et d'un bon recours pour la mise à disposition, suivant les conditions écologiques, d'équations plus appropriées pour l'estimation du volume des arbres des essences des écosystèmes forestiers. Sur le plan juridique, cette méthodologie vient renforcer l'application non seulement des lois, textes législatifs et réglementaires forestiers, mais aussi des conventions internationales comme la convention sur la biodiversité et la convention sur la désertification.

\section{Références bibliographiques}

AKINSANMI F. A., AKINDÉLÉ S. O., 1995. Teak productivity in relation to soil conditions : A re-assessment of teak plantations in the dry high forest area of south-western Nigeria. Nigerian Journal of Forestry, 24 : 7-10.

DAWKINS H. C., 1961. Estimating total volume of some Caribbean trees. Caribbean Forestry, 22 (3-4) : 62-63.

FONTON N. H., GLELE KAKAÏ R., RONDEUX J., 2002. Étude dendrométrique de Acacia auriculiformis A. Cunn ex Benth en mélange sur vertisol au Bénin. Biotechnologie, Agronomie, Société et Environnement, 6 (1) : 29-37.

LAUMANS P., 1991. Tables de cubage à deux entrées pour le volume bois sciable du Teck (Tectona grandis L. f. ) au Sud Bénin. Wittelsbacherstr 11, D-8016 Feldkirchen, 18 p.

PALM R., 1982. Influence de la formule de cubage et de la longueur des billons sur la détermination du volume des arbres abattus. Annales des Sciences Forestières, 39 : 231-238.

PALM R., IEMMA A. F., 2002. Conditions d'application et transformations de variables en régression linéaire. Gembloux, Belgique, Faculté universitaire des sciences agronomiques, Notes de Statistique et d'Informatique, $34 \mathrm{p}$.

PAUWELS D., RONDEUX J., 1999. Tarifs de cubage pour les petits bois de melèze (Larix sp.) en Ardenne. Les Cahiers Forestiers de Gembloux, 11p.

PHILIP S. M., 1998. Measuring trees and forests. $2^{\text {nd }}$ ed. Wallingford, Royaume-Uni, CABI, $310 \mathrm{p}$.

RONDEUX J., 1999. La mesure des arbres et des peuplements forestiers. Gembloux, Belgique, Les Presses Agronomiques de Gembloux, $2^{\mathrm{e}}$ édition, $521 \mathrm{p}$.

THALMANN A., 1965. À propos des tarifs de cubage. Bois et Forêts des Tropiques, $101:$ 29-30.

THIBAUT A., RONDEUX J., CLAESSENS H., 1998. Tarifs de cubage pour l'Aulne glutineux [Alnus glutineux (L.) Gaertn.] en Belgique méridionale. Revue Forestière Française, L-3 : 244-250. 